\title{
Mathematical Models of Coverage Areas in the Earth Plane for Video and Radar Images
}

\author{
Anatoly I. Novikov, Sergey A. Yukin, Alexander A. Loginov, Michael B. \\ Nikiforov, Yevgeniy R. Muratov, Victoria A. Sablina \\ Department of Higher Mathematics and Department of Electronic Computers, RSREU, Ryazan, \\ 390005, Russia
}

Keywords: the superimposition, radar images of the underlying surface, the area of the ambiguity.

\begin{abstract}
The mathematical models of the coverage areas in the Earth plane are suggested. These models correspond to images obtained from both video sources and an on-board radio station working in the forward hemisphere. The aircraft navigation parameters serve as input data to construct the above-stated areas. There are six parameters which describe spatial and angular aircraft coordinates. The geometry of the coverage areas is investigated for the two cases. At first the ideal conditions without errors of navigation parameters are considered. Then the conditions with different errors of navigation parameters are also considered. It is shown that it is necessary to enlarge the search area for the tasks of the cartographic information superimposition with the aerial video and radar images. This enlargement should be done taking into account the area of the ambiguity which is generated for the most unfavorable combination of the errors of navigation parameters.
\end{abstract}

\section{Introduction}

The modern aircrafts are equipped with various on-board computer vision systems [1]. These systems work not only in different spectral ranges but on different principles. They can process information from infrared imagers, video cameras, a LiDAR and radar. The variety of sensors leads to the importance of the integration task for this information. To solve this task on board of the aircraft it is necessary to bring all the information to the comparable form for the subsequent superimposition [2]. Such superimposition tasks emerge in the different technical fields [3]. It is impossible to superimpose heterogeneous images directly, e.g. by the form features, so indirect methods are required to match the images from the different sensors and the digital terrain map.

The images generated by the aircraft route and the map can't be superimposed at once. The reason for this is the aircraft navigation system errors. So it is necessary among other things to correct geometric distortions arose from the measurement uncertainty of the navigation system. It will give a chance to perform the superimposition automatically. When superimposing heterogeneous images, the strict requirements to the superimposition accuracy should be met [4].

The aerial photograph of the corresponding area of the underlying surface and its analog synthesized from the map can't be superimposed without information about the sizes of the image area in the Earth plane. The ambiguity of the coverage area in the Earth plane arises because of the errors in the navigation parameters values. The synthesized image area can be generated from the digital terrain map using the current values of the vector $\mathbf{v}=(x, y, h, \psi, \theta, \gamma)$ of the navigation parameters. However, the generated synthesized image will not match with the observed aerial photograph because of the errors $\Delta \boldsymbol{v}=(\Delta x, \Delta y, \Delta h, \Delta \psi, \Delta \theta, \Delta \gamma)$ of the navigation parameters [5]. Therefore, it is necessary to determine the limiting area, or the area of the ambiguity, of the synthesized image taking into consideration the limiting values of the errors of the navigation parameters.

An example of the synthesized map image superimposition with the underlying surface video image is represented in Fig. 1. The synthesized image is generated from the digital terrain map using the navigation parameters. It consists of the contours of the main objects, viz. the water 
objects. The simple imposition of the images in Fig. 1 reveals the significant mismatch of the contours of the main objects. This mismatch is caused by the errors of the navigation parameters.

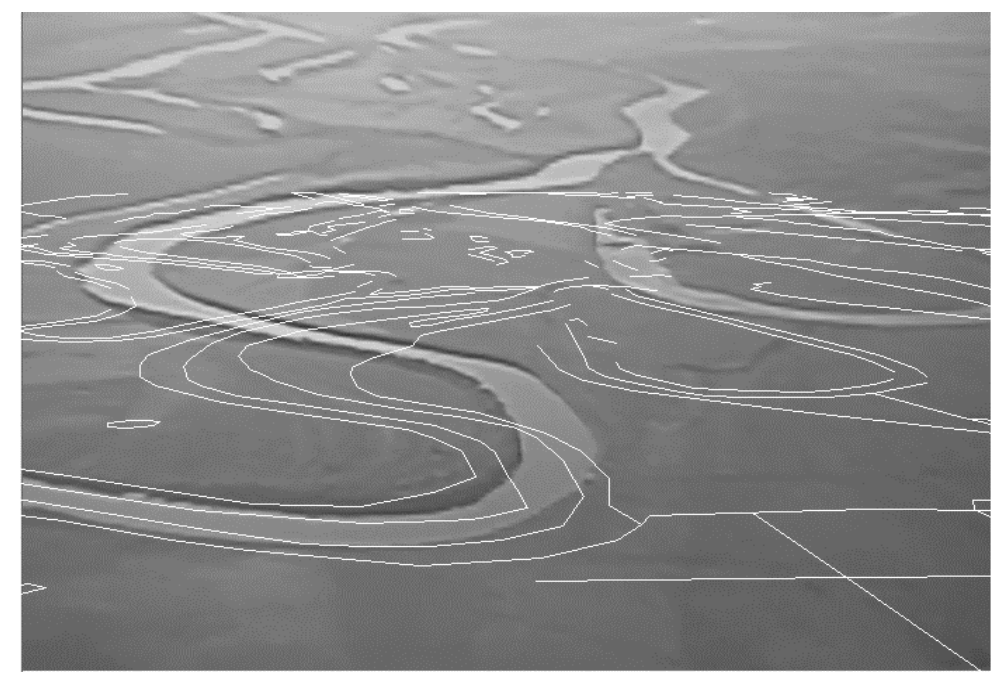

Fig.1: The result of the simple synthesized image (light lines) imposition on the aerial photograph

\section{The Mathematical Model for A Video Image}

Let us put into consideration the rectangular coordinate system Oxyz. And let us determine this system in the following way. Its origin coincides with the aircraft center of mass. The axis $O y$ goes along the aircraft construction line. The axis $O x$ is perpendicular to the axis $O y$ and goes along the aircraft right wing. The axis $O z$ is perpendicular to the plane $O x y$. The plane $O y z$ in the constructed coordinate system is the pitch $(\theta)$ plane. The plane $O x y$ is the yaw $(\psi)$ plane. The plane $O x z$ is the roll $(\gamma)$ plane.

The video camera field of view represents the pyramid with the vertex coincided with the camera location point. As a first approximation, the origin of the considered coordinate system can be assumed as this point. The base of the pyramid is the coverage area in the Earth plane. It is the convex quadrangle $A_{1} B_{1} B_{2} A_{2}$ in Fig. 2.

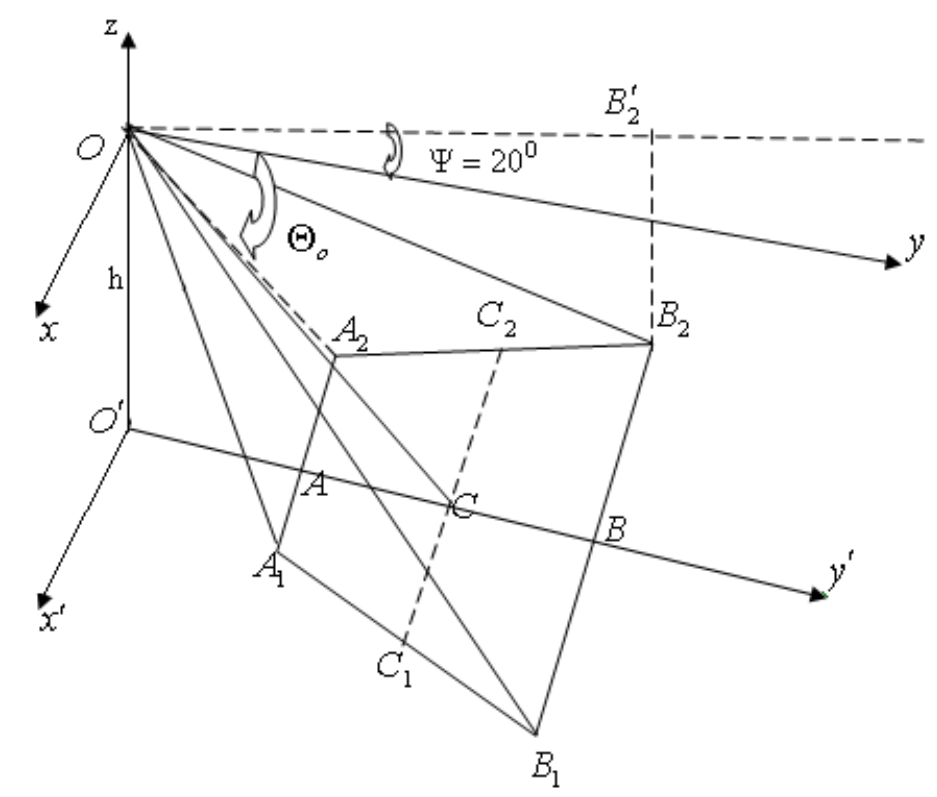

Fig.2: The coverage area $A_{1} B_{1} B_{2} A_{2}$ in the Earth plane without errors 
The on-board aircraft video camera is mounted in a real system at the certain angle to the axis $O y$ in the pitch plane. This angle is chosen to make the camera angle of view in this plane a few degrees above the horizon line. It is $9^{\circ}$ for the civil aircraft with $40^{\circ} \times 30^{\circ}$ camera angle of view. Here $40^{\circ}$ is the angle in the yaw plane. And $30^{\circ}$ is the angle in the pitch plane. Therefore the coverage area in the Earth plane in the actual condition is unlimited. Fig. 2 represents the case when the upper face of the viewing pyramid is located below the plane $O x y$.

To construct the coverage view in the Earth plane taking into account the determination errors of the aircraft location in the space let us implement the following algorithm [6].

1. The unit vectors of $\overline{O A_{1}}, \overline{O B_{1}}, \overline{O B_{2}}, \overline{O A_{2}}$ are found.

2. The rotation of this vector system in the yaw, pitch and roll planes at the specified angles is performed. As a result, the new unit vectors $\overline{O A_{1}^{\prime}}, \overline{O B_{1}^{\prime}}, \overline{O B_{2}^{\prime}}, \overline{O A_{2}^{\prime}}$ can be obtained.

3. The equations of the lines passing through the point $O$ are composed. The point $O$ is the origin of the moving coordinate system where the aircraft is located. And then the coordinates of the points $A_{1}^{\prime}, B_{1}^{\prime}, B_{2}^{\prime}, A_{2}^{\prime}$ of the intersection of these lines and the Earth plane can be found. It is the coordinates of the corner points of the coverage area constructed taking into consideration the angular errors.

Let us find the unit vectors $\overline{O A_{1}}, \overline{O B_{1}}, \overline{O B_{2}}, \overline{O A_{2}}$ by the standard formula $\bar{q}^{0}=\bar{q} /|\bar{q}|$. Then let us form the 4-by-3 matrix $Q$ from the coordinates of the found unit vectors $\bar{q}_{i}^{0}$. To find the unit vectors of $\overline{O A_{1}^{\prime}}, \overline{O B_{1}^{\prime}}, \overline{O B_{2}^{\prime}}, \overline{O A_{2}^{\prime}}$ it is necessary to multiply the matrix $Q$ by the matrix $T(\Delta \gamma, \Delta \theta, \Delta \psi)=T_{\Delta \gamma} \cdot T_{\Delta \theta} \cdot T_{\Delta \psi}$. The latter matrix implements the rotations of the unit vectors $\bar{q}_{i}, i=\overline{1,4}$ of the angles $\Delta \psi, \Delta \theta, \Delta \gamma$ in the yaw, pitch and roll planes respectively. The rotation matrices $T_{\Delta \psi}, T_{\Delta \theta}, T_{\Delta \gamma}$ are of the following form:

$$
T_{\Delta \psi}=\left(\begin{array}{ccc}
\cos \Delta \psi & -\sin \Delta \psi & 0 \\
\sin \Delta \psi & \cos \Delta \psi & 0 \\
0 & 0 & 1
\end{array}\right), T_{\Delta \theta}=\left(\begin{array}{ccc}
\cos \Delta \theta & 0 & \sin \Delta \theta \\
0 & 1 & 0 \\
-\sin \Delta \theta & 0 & \cos \Delta \theta
\end{array}\right), T_{\Delta \gamma}=\left(\begin{array}{ccc}
1 & 0 & 0 \\
0 & \cos \Delta \gamma & -\sin \Delta \gamma \\
0 & \sin \Delta \gamma & \cos \Delta \gamma
\end{array}\right),
$$

The last step of the algorithm is the estimation of the coordinates of the coverage area corner points in the Earth plane taking into account the angular errors. The coordinates $x_{i}^{\prime}=-h \frac{l_{i}^{\prime}}{n^{\prime}}$, $y_{i}^{\prime}=-h \frac{m_{i}^{\prime}}{n_{i}^{\prime}}$ of the $i$-th point in the Earth plane can be found from the equations of the lines $O A_{1}^{\prime}, O B_{1}^{\prime}, O B_{2}^{\prime}, O A_{2}^{\prime}: L_{i}: \frac{x_{i}^{\prime}}{l_{i}^{\prime}}=\frac{y_{i}^{\prime}}{m_{i}^{\prime}}=\frac{z_{i}^{\prime}}{n_{i}^{\prime}}, \quad i=\overline{1,4}$ passing through the point $O$ where the aircraft is located and the equation of the Earth plane $z=-h$.

The coverage areas in the Earth plane for the video images without the navigation errors and with the angular errors of the navigation parameters are represented in Fig. 3. 


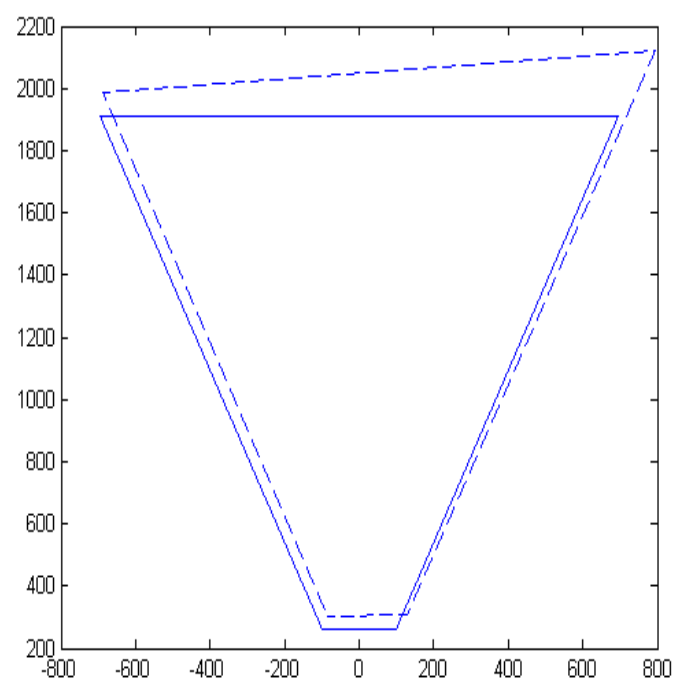

Fig.3: The video coverage areas in the Earth plane without errors (the solid line) and with the angular errors $\Delta \psi=0,2^{\circ}, \Delta \theta=0,1^{\circ}, \Delta \gamma=0,3^{\circ}$ (the dash line)

Let us consider the following parameters of the obtained areas for the comparison.

The lengths of the segments $A_{1} A_{2}$ and $A_{1}^{\prime} A_{2}^{\prime}$ are $196.2 \mathrm{~m}$ and $214.9 \mathrm{~m}$ (the latter is longer on $9.5 \%$ ). The lengths of the segments $B_{1} B_{2}$ and $B_{1}^{\prime} B_{2}^{\prime \prime}$ are $1389.0 \mathrm{~m}$ and $1488.0 \mathrm{~m}$ (the latter is longer on $7.1 \%$ ). The lengths of the segments $A_{1} B_{1}$ and $A_{1}^{\prime} B_{1}^{\prime}$ are $1752.2 \mathrm{~m}$ and $1785.5 \mathrm{~m}$ (the latter is longer on $1.9 \%$ ). The lengths of the segments $A_{2} B_{2}$ and $A_{2}^{\prime} B_{2}^{\prime}$ are $1752.2 \mathrm{~m}$ and $1935.0 \mathrm{~m}$ (the latter is longer on $10.4 \%$ ).

The area outlined by the solid line in Fig. 3 is $1.31 \mathrm{~km}^{2}$. The area obtained with errors and outlined by the dash line in Fig. 3 is $1.48 \mathrm{~km}^{2}$. The latter is larger than the first area on the $13 \%$.

The comparative analysis of the parameters given above indicates the significant changes in the geometry and the sizes of the coverage area in the Earth plane. In this case it is caused only by the determination errors of the aircraft yaw, pitch and roll. The determination errors of the aircraft altitude and current coordinates change the coverage area in the Earth plane to an even greater degree especially at low altitude.

\section{The Mathematical Model for A Radar Image}

The algorithm for the coverage area generation in the Earth plane for a radar image for the most part agrees with the algorithm given above. However, it also has some fundamental differences.

Let us assume the following. The radar range is not greater than the maximum slant range. This range is considered as the range from the radar of the aircraft to the underlying surface when the tilt angle of the upper bound of the radiation pattern is $\beta_{v}=5^{\circ}$. Let us denote this quantity by $R_{\max }$. The minimum slant range is considered as the range from the radar of the aircraft to the underlying surface when the tilt angle of the lower bound of the radiation pattern is $\beta_{n}=35^{\circ}$. Let us denote this quantity by $R_{\min }$. The considered quantities are represented in Fig. 4. 


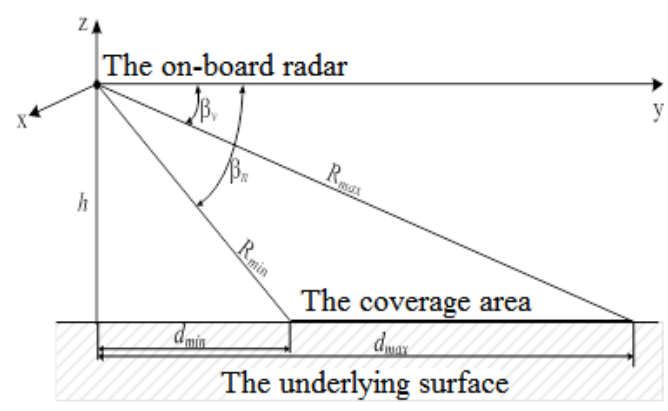

Fig.4: The generated coverage area relative to the aircraft

The distances $d_{\max }, d_{\min }$ are the distances to the near and far edges of the coverage area in the Earth plane. The quantity $d$ is the length of this area. These quantities can be calculated by the following formulas:

$$
d_{\text {max }}=R_{\text {max }} \cdot \cos \beta_{v}, \quad d_{\text {min }}=R_{\min } \cdot \cos \beta_{n}, \quad d=d_{\text {max }}-d_{\text {min }} .
$$

The coverage area in the Earth plane is of the form of the truncated circular sector $A_{1} B_{1} B_{2} A_{2}$ when $\alpha_{0}=0$, i.e., when the center of the radar view coincides with the aircraft axis, and when there are no determination errors of the navigation parameters. It is shown in Fig. 5.

The coordinates of the corner points $A_{1}, A_{2}, B_{1}, B_{2}$ of the coverage area in the Earth plane under the constraints stated above can be calculated by the following formulas:

$$
\begin{aligned}
& x_{A 1}=d_{\min } \sin \frac{\alpha}{2}, \quad y_{A 1}=d_{\min } \cos \frac{\alpha}{2}, x_{A 2}=-x_{A 1}, y_{A 2}=y_{A 1} ; \\
& x_{B 1}=d_{\text {max }} \sin \frac{\alpha}{2}, \quad y_{B 1}=d_{\text {max }} \cos \frac{\alpha}{2} ; x_{B 2}=-x_{B 1}, y_{B 2}=y_{B 1} .
\end{aligned}
$$

Let us generate from them the 4-by-2 matrix $X$. Further the described above algorithm for the coverage area generation in the Earth plane for a video image is implemented. The important addition is included in this algorithm in the case of the range strobing.

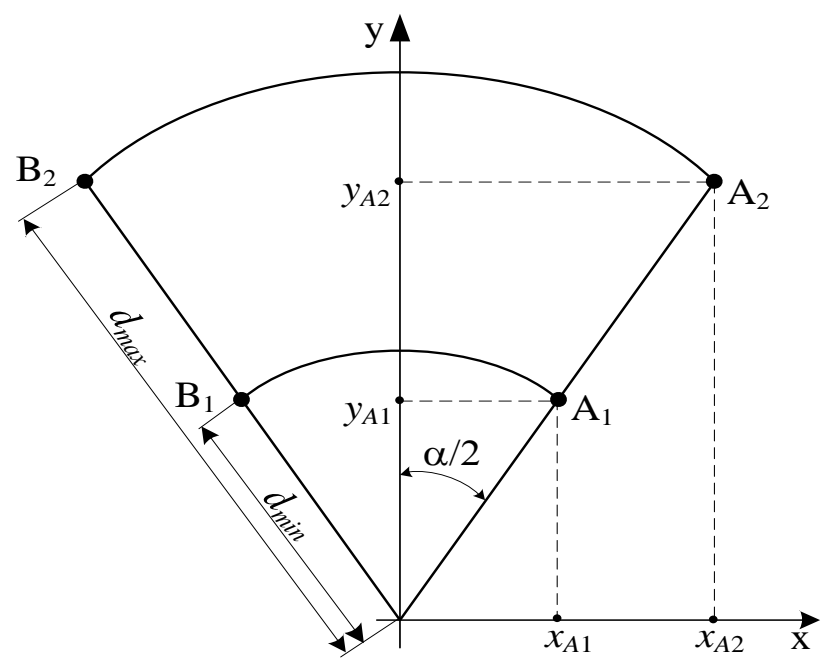

Fig.5: The form of the coverage area in the Earth plane

An example of the radar coverage area in the Earth plane in the presence of the angular errors and with no account taken of the range strobing is represented in Fig. 6. 


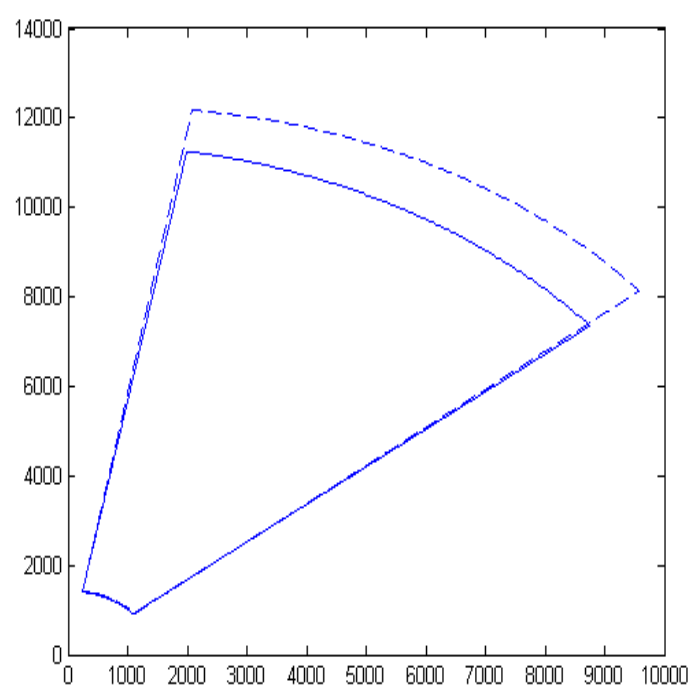

Fig.6: The radar coverage areas in the Earth plane without errors (the solid line) and with the angular errors $\Delta \psi=\Delta \theta=\Delta \gamma=0,32^{\circ}$ (the dash line)

\section{Conclusions}

The analysis of the coverage areas in the Earth plane using the suggested mathematical models makes it possible to draw the following conclusions. The distortions of the video camera coverage area can be very significant especially at low altitude. It can occur when the combination of the aircraft position errors $\Delta x, \Delta y, \Delta h$ and the aircraft orientation errors $\Delta \psi, \Delta \theta, \Delta \gamma$ is unfavorable. The distortions of the radar coverage area in the Earth plane can reach from 100 to $135 \mathrm{~m}$ with given parameters of the radar and the navigation system. Here the distortions are measured in the deviations of the corner points relative to the undistorted image. This corresponds with approximately the $1 \%$ of the maximum horizontal distance $d_{\max }$ and the $7 \%$ of the minimum horizontal distance $d_{\min }$.

The coverage areas for the sensors working in different spectral ranges and on different principles can be distorted in different ways. It is necessary to take into consideration these potential distortions when generating combined images and also when superimposing a synthesized map image with an aerial photograph.

\section{References}

[1] David A. Forsyth and Jean Ponce, Computers Vision: A Modern Approach, 2nd ed., New York, Prentice-Hall, 2011, 792 p.

[2] Victoria A. Sablina, Anatoly I. Novikov, Michael B. Nikiforov, and Alexander A. Loginov, An Approach to the Image Superimposition Problem in Multispectral Computer Vision Systems, 2nd Mediterranean Conference on Embedded Computing (MECO), Budva, Montenegro, 2013, pp.117-120.

[3] Lu Wang, Ulrich Neumann, A robust approach for automatic registration of aerial images with untextured aerial LiDAR data, Computer Vision and Pattern Recognition (CVPR), Miami, FL, USA, 2009, pp. 2623-2630.

[4] DO-315B Minimum Aviation System Performance Standards (MASPS) for Enhanced Vision Systems, Synthetic Vision Systems, Combined Vision Systems and Enhanced Flight Vision Systems, 2011. 
[5] Victoria A. Sablina, Anatoly I. Novikov, Michael B. Nikiforov, and Alexander A. Loginov, Navigation Parameters Correction Technique Using Multiple View Geometry Methods, 22nd International Conference in Central Europe on Computer Graphics, Visualization and Computer Vision (WSCG) Communication Paper Proceedings. University of West Bohemia, Plzen, Czech Republic, 2014, pp. 103-110.

[6] E.V. German, E.R. Muratov, A.I. Novikov, Mathematical Model of Uncertainty Zone in Combined Image Problem // Bulletin of the Ryazan State Radio Engineering University. Ryazan, Russia, 2013. Volume 46, Issue 4, Part 2, pp. 11-16. (In Russian). 